\section{Programa Práticas Educativas em Segurança dos Alimentos na Cidade Universitária Armando de Salles Oliveira (CUASO-USP)}

\author{
Educational Practices in Food Safety Program on University \\ City Armando de Salles Oliveira (CUASO-USP)
}

\section{RESUMO}

Em 2008 a Universidade de São Paulo (USP) sediou a primeira edição do Fórum Permanente sobre Espaço Público que trouxe como um dos temas de interesse da comunidade USP a "segurança dos alimentos comercializados no campus". Em 2010 a Faculdade de Medicina Veterinária e Zootecnia da USP estruturou o Programa Práticas Educativas em Segurança dos Alimentos (PESA), trabalho integrado à Prefeitura do Campus da Universidade de São Paulo, na capital. Até o primeiro semestre de 2012, o Programa PESA identificou e cadastrou 53 pontos de venda de alimentos no campus, os quais comercializam, a cada mês, cerca de 40250 refeições, 11580 lanches e 23 mil salgados e pastéis. Esses estabelecimentos já passaram por uma primeira avaliação de boas práticas até o final de 2011 e se encontram na segunda fase de avaliação e orientação técnica para a correção de não conformidades. Além de visitas técnicas de avaliação de boas práticas, o Programa PESA promove Reuniões Técnicas com os comerciantes de alimentos do campus e Encontros Técnicos de Formação da Equipe de Trabalho. O programa é uma iniciativa com proposta totalmente inovadora na Cidade Universitária Armando de Salles Oliveira, inexistente em outros campi e em outras universidades brasileiras e representa para graduandos e pós-graduandos um inovador espaço de contato direto com realidades concretas, de desenvolvimento de habilidades e de aplicação de conhecimentos numa interação de saberes acadêmicos e populares.

Palavras-chave: Práticas educativas. Segurança dos alimentos. Universidade de São Paulo.

\section{ABSTRACT}

In 2008 the University of São Paulo (USP) hosted the Public Space Permanent Forum first edition that brought the topic 'safety of food sold on campus' as one of the USP

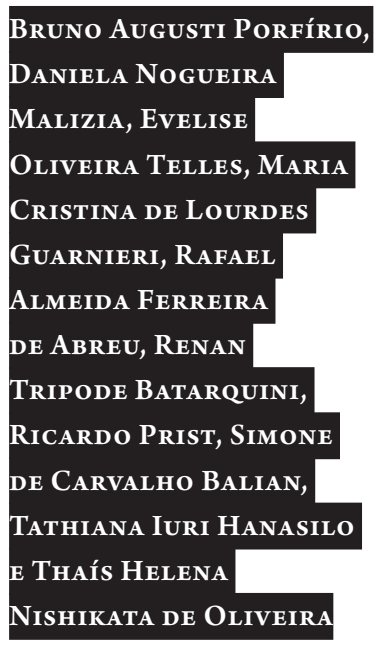


community interest topics. In 2010 the Faculty of Veterinary Medicine of USP structured the Educational Practices in Food Safety Program, an integrated work with the USP Capital Campus Hall. Until the half of 2012 the Program has identified and registered 53 food sale points on campus, which sell approximately 40,250 meals, 11,580 sandwiches and 23,000 snacks and pastries each month. These establishments have gone through an initial assessment of good practice by the end of 2011 and are on the second phase of evaluation and technical guidance to correct noncompliance. In addition to technical visits for evaluation of good practices, the Program promotes technical meetings with the campus food traders and Technical Training Meetings of the Task Force. The program is a totally new initiative proposed in the University City Armando de Salles Oliveira, absent from other campi and other Brazilian universities and represents to undergraduates and graduate students an innovative space for direct contact with concrete realities, skills development and application of knowledge on the interaction of academic and popular knowledge.

Keywords (Keywords): Educational practices. Food safety. University of São Paulo.

\section{INTRODUÇÃO}

A Cidade Universitária Armando de Salles Oliveira (CUASO-USP) se caracteriza como uma cidade de porte médio, na qual circulam, aproximadamente, 100 mil pessoas por dia [11].

No decorrer do ano de 2008, a USP sediou a primeira edição do Fórum Permanente sobre Espaço Público, iniciativa da Prefeitura do Campus USP da Capital (PUSP-C), que permitiu a discussão entre as instâncias de gestão e usuários do campus, da prática da cidadania coletiva. Um dos temas de interesse foi "a Segurança dos Alimentos comercializados na CUASO-USP”. Entre as propostas elaboradas pelos participantes do Fórum, teve destaque o ponto sobre a qualidade dos alimentos preparados e comercializados no local, apontando questões como: política de alimentação por áreas do campus; implementação de "praças de alimentação", inovações tecnológicas para a sustentabilidade, entre outras. No final de 2009 a PUSP-C implementou o "Programa Campus Sustentável”, constituído por três eixos:

1. Infraestrutura Adequada para a Sustentabilidade;

2. Qualidade de Vida a partir de ações sustentáveis;

3. Gestão Participativa/Transparência.

Em 2010 a Faculdade de Medicina Veterinária e Zootecnia da USP (FMVZ-USP) iniciou a sua participação no eixo "Qualidade de Vida” com a estruturação do Programa PESA (Práticas Educativas em Segurança dos Alimentos), que compreende um conjunto de ações transformadoras das relações entre pessoas e o meio ambiente, num contexto entre Alimentação - Saúde - Qualidade de Vida. A proposta vem oferecer um espaço inovador para a aplicação de conhecimentos teóricos e desenvolvimento de habilidades de graduandos, desenvolvimento de pesquisas em pós-graduação e extensão de conhecimentos e prestação de serviços à comunidade no tema higiene e segurança dos alimentos. 
Nesse contexto o Programa PESA tem por objetivos:

1. Caracterizar o cenário da alimentação no campus;

2. Cadastrar os estabelecimentos que comercializam alimentos;

3. Cesenvolver instrumentos de monitoramento das boas práticas de higiene;

4. Realizar visitas técnicas com caráter de orientação aos comerciantes de alimentos;

5. Criar um espaço de aplicação de conhecimentos para os graduandos.

O presente Programa traz a oportunidade de gradativamente incrementar a segurança dos alimentos comercializados no campus e a gestão dos resíduos gerados, criando um espaço de interação dos atores Estudantes - Docentes - Usuários - Comerciantes de Alimentos Gestores do campus, onde todos estão focados na sustentabilidade socioambiental.

\section{MATERIAIS E MÉTODOS}

O campus da CUASO-USP está estruturado por um sistema de localização espacial baseado nas cores azul, verde, amarelo e vermelho de modo a facilitar a administração e a geolocalização de seus institutos, unidades, vias públicas e estabelecimentos comerciais [1]. Os estabelecimentos que comercializam alimentos estão classificados em RL (Restaurantes/Lanchonete) e PE (Ponto de Venda Externo de Alimentos - carrinhos de hot dog, pipoca, quiosques de alimentação), a partir de critérios como porte, complexidade de processos e tipos de alimentos comercializados. A metodologia proposta para condução deste trabalho baseia-se na pesquisa-ação com atividades participativas e interativas dos graduandos, pós-graduandos, docentes e funcionários com os comerciantes, responsáveis técnicos e manipuladores de alimentos no campus [3].

Os estabelecimentos devem cumprir boas práticas de higiene e manipulação de alimentos, em conformidade com: a Resolução Federal 216 [8], o Código Sanitário Estadual [5], a Portaria Estadual CVS 6/99 [9], o Código Sanitário Municipal [6] e a Portaria no 2.619/2011 da Secretaria Municipal de Saúde de São Paulo [10]. Utiliza-se também o Manual de Boas Práticas de Manipulação de Alimentos [7]. Considera-se não conforme todas as práticas, procedimentos e situações em desacordo à legislação vigente. As Listas de Verificação utilizadas pela equipe compõem-se de três eixos fundamentais:

1. Higiene e Manipulação de Alimentos;

2. Tempo e Temperatura nas Fases de Produção;

3. Limpeza e Desinfecção de Utensílios.

Nas reuniões semanais a equipe discute as não conformidades observadas e registradas nas visitas técnicas com docentes. A partir daí, elaboram-se os relatórios no banco de dados, sendo então gerados os Relatórios de Visita Técnica. Após a Visita Técnica, volta-se ao estabelecimento, discute-se com o proprietário ou responsável técnico o que deve ser feito para o incremento das BPHM e estabelecem-se prazos para as adequações. A PUSP-C fica encarregada de encaminhar os Relatórios de Visita Técnica para 
as Unidades e os Institutos aos quais pertence cada um dos estabelecimentos.

A partir de 2011 adotou-se a realização de encontros técnicos mensais congregando graduandos, pós-graduandos e os professores/pesquisadores, para o aprimoramento continuado da equipe, convidando profissionais para discorrer sobre os temas técnicos específicos e relevantes no contexto do projeto. Até julho de 2012 foram abordados os temas: Manejo de Resíduos, Vigilância Sanitária de Alimentos, Segurança do Trabalho, Higiene e Segurança Alimentar, Gestão de Pessoas, Frio Alimentar, Atendimento ao Cliente. Todas as ações dos graduandos e pós-graduandos são coordenadas por um docente da FMVZ-USP e por um gestor da PUSP-C, mantendo-se reuniões semanais para discussão das ações realizadas e estruturação dos próximos trabalhos.

\section{RESULTADOS}

Desde o início do Programa (2010) participaram quatro graduandos em Medicina Veterinária; um graduando em Ciência da Computação; quatro médicos veterinários formados; dois pós-graduandos em Epidemiologia Experimental e Aplicada às Zoonoses e dois docentes da FMVZ-USP. Foram realizadas três Reuniões Técnicas com os comerciantes de alimentos da CUASO (para apresentação do Programa e exposição de resultados) e sete Encontros Técnicos de Formação da Equipe de Trabalho, com temas: Frio Alimentar; Vigilância Sanitária de Alimentos; Segurança do Trabalho (parte 1 e 2); Sistemas de Autocontrole Higiênico e Sanitário de Alimentos; Gestão de Pessoas e Gestão de Resíduos.

A equipe de trabalho já elaborou os seguintes documentos:

1. Guia de Orientação no uso das Listas de Avaliação de BPHM;

2. Listas de Verificação;

3. Relatório de Visita Técnica;

4. Relatório de Retorno;

5. Material Didático de Orientação para Lavagem de Mãos.

Até o primeiro semestre de 2012, o Programa PESA identificou e cadastrou na CUASO-USP 53 pontos de venda de alimentos (Figura 1), nos quais são comercializados cerca de 40250 refeições, 11580 lanches e 23 mil salgados, mensalmente. Em média, o tempo de atuação dos comerciantes de alimentos no campus verificado foi de onze anos, sendo que o mais antigo atua no campus desde 1974, e os mais recentes foram instalados em 2011.

Dos 53 estabelecimentos cadastrados, seis atuam nos finais de semana, 33 preparam pelo menos um tipo de alimento no local, 50 citaram ter pelo menos um dos produtos sendo recebido em veículo de fornecedor autorizado e refrigerado, sendo que os demais transportam todos os produtos em veículo próprio. Verificou-se uma maior concentração de pontos de venda de alimentos no setor Azul (34\%), seguido pelos setores Verde (32\%), Amarelo (20,8\%) e Vermelho (13,2\%) da CUASO (Gráfico 1). 
Gráfico 1 Perfil geral de distribuição dos pontos de venda de alimentos cadastrados na Cidade Universitária Armando de Salles Oliveira

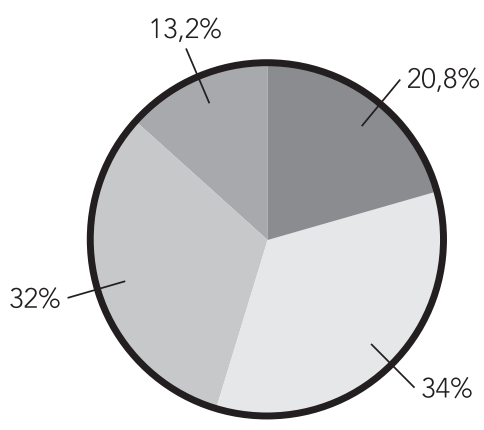

Setor Azul

Setor Verde

Setor Vermelho

Setor Amarelo

Esses estabelecimentos já passaram por uma primeira avaliação de boas práticas até o final de 2011 e encontram-se na segunda fase de avaliação e orientações técnicas em boas práticas e adequação das não conformidades.

Os tipos de comércio mais prevalentes verificados no campus (Gráfico 2) foram os Restaurantes/Lanchonetes (38\%) e Lanchonetes (38\%), seguidos pelos Pontos Externos de venda (19\%) e outros tipos de comércio, a saber, açougue, mercado e padaria (5\%). Os Pontos Externos de venda são representados pelos carrinhos e quiosques de alimentação que utilizam apenas as dependências externas das instituições do campus.

Os comércios do tipo Lanchonete foram identificados em maior número apenas no Setor Vermelho (43\%), sendo o tipo misto Restaurante/Lanchonete mais frequente nos setores Azul (45\%) e Amarelo (55\%), e os Pontos Externos (ambulantes e quiosques) mais comuns no Setor Verde (41\%) (Tabela 1).

Todos os 53 estabelecimentos cadastrados utilizam a coleta de lixo do campus; entretanto, onze deles não possuem pontos de água e cinco não possuem pontos de energia elétrica.

Foram considerados pelos comerciantes, como pontos positivos em atuar na CUASO a "segurança", a "clientela educada e fixa" e o "contato com outras pessoas". Foram considerados como pontos negativos os "processos licitatórios", as "greves" e as "questões relacionadas à infraestrutura de prédios e instalações”.

Verificou-se através das visitas técnicas aos estabelecimentos que $51 \%$ das não conformidades estavam relacionadas ao eixo Higiene e Manipulação de Alimentos, 34\% ao eixo Tempo e Temperatura nas Fases de Produção e 15\% ao eixo Limpeza e Desinfecção de Utensílios (Gráfico 3 ). 
Gráfico 2 Distribuição dos estabelecimentos segundo o tipo de comércio alimentício

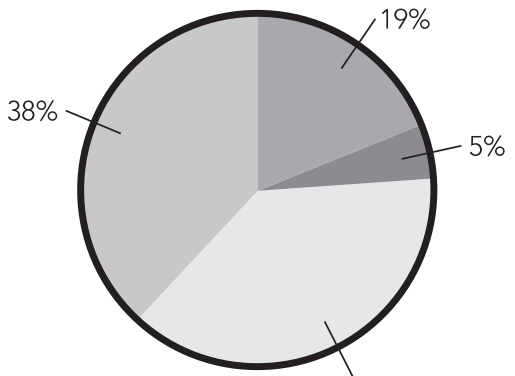

$38 \%$

Restaurante/Lanchonete

Lanchonete

Ponto Externo

Outros (açougue, mercado e padaria)

Tabela 1 Distribuição dos estabelecimentos segundo o tipo de comércio alimentício verificado em cada setor da Cidade Universitária Armando de Salles Oliveira

\begin{tabular}{lcccc}
\hline & $\begin{array}{c}\text { SETOR } \\
\text { AZUL }\end{array}$ & $\begin{array}{c}\text { SETOR } \\
\text { VERDE }\end{array}$ & $\begin{array}{c}\text { SETOR } \\
\text { AMARELO }\end{array}$ & $\begin{array}{c}\text { SETOR } \\
\text { VERMELHO }\end{array}$ \\
\hline Restaurante/Lanchonete & $45 \%(8 / 18)$ & $24 \%(4 / 17)$ & $55 \%(6 / 11)$ & $29 \%(2 / 7)$ \\
\hline Lanchonete & $40 \%(7 / 18)$ & $35 \%(6 / 17)$ & $36 \%(4 / 11)$ & $43 \%(3 / 7)$ \\
\hline Ponto Externo & $5 \%(1 / 18)$ & $41 \%(7 / 17)$ & $9 \%(1 / 11)$ & $14 \%(1 / 7)$ \\
\hline Padaria & - & - & - & $14 \%(1 / 7)$ \\
\hline Açougue & $5 \%(1 / 18)$ & - & - & - \\
\hline Mercado & $5 \%(1 / 18)$ & - & 11 & - \\
\hline No de estabelecimentos & 18 & 17 & & \\
cadastrados (53) & & & & - \\
\hline$-:$ não foram cadastrados estabelecimentos nessa categoria & & & \\
\hline
\end{tabular}


Gráfico 3 Distribuição das não conformidades verificadas em visitas técnicas aos estabelecimentos segundo cada eixo de avaliação de boas práticas de higiene e manipulação de alimentos. Total de não conformidades: 18/53 estabelecimentos.

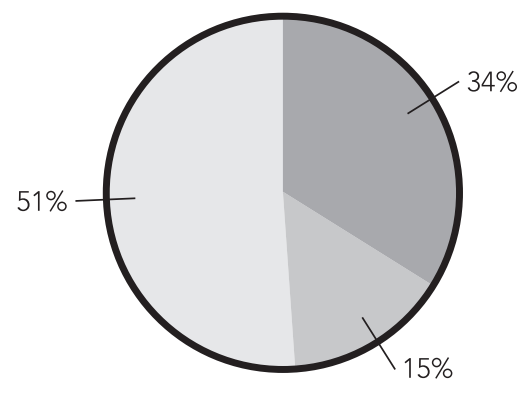

Higiene e manipulação de alimentos

$\square$ Tempo e temperatura

Limpeza e desinfecção de alimentos

\section{DISCUSSÃO}

É fundamental ressaltar que esta é uma iniciativa totalmente inovadora na CUASO-USP, pois trata-se da primeira vez, na história da Universidade de São Paulo, que se procura reconhecer e documentar a existência desse serviço. Vale lembrar também que inexistem estudos similares em outros campi e outras universidades brasileiras. Além de ser um trabalho que integra ensino, pesquisa e extensão universitária, abrindo oportunidades de aplicação dos conhecimentos acadêmicos, praticando a cidadania coletiva, é uma iniciativa que vem ao encontro da definição de Segurança Alimentar e Nutricional estabelecida no Brasil, em 2004 pelo Conselho Nacional de Segurança Alimentar e Nutricional (CONSEA), que determina que:

[...] todos os indivíduos têm o direito ao acesso regular e permanente a alimentos de qualidade, em quantidade suficiente, sem comprometer o acesso a outras necessidades essenciais à sua vida, tendo por base, práticas alimentares que promovem a saúde, que respeitam a diversidade cultural e que sejam social, econômica e ambientalmente sustentáveis [...]. [2]

Neste cenário, a Faculdade de Medicina Veterinária e Zootecnia da USP, em parceria com a PUSP-C, inova no contexto ensino - aprendizagem ao promover a participação de graduandos, docentes e pesquisadores com o programa institucional "Práticas Educativas em Segurança dos Alimentos na CUASO-USP” que possibilita:

1. A melhoria da educação no ensino superior pautada na cidadania e função social;

2. A oportunidade do contato direto de estudantes com realidades concretas praticando 
a troca de saberes acadêmicos e populares.

No que se refere às questões técnicas da segurança dos alimentos, fica evidente a necessidade de continuidade do trabalho, levando esclarecimentos e melhorias técnicos aos pontos de venda de alimentos, considerando as seguintes falhas identificadas: 51\% quanto à higiene e manipulação; 34\% quanto a controle de tempo e temperatura de processos e comercialização de alimentos e $15 \%$ quanto ao controle da limpeza e desinfecção dos utensílios e ambientes.

O Programa PESA tem por missão incrementar melhorias na segurança dos alimentos e na qualidade dos serviços na CUASO-USP através das vivências e troca de conhecimentos entre seus atores.

\section{CONCLUSÕES}

O Programa PESA representa, para graduandos e pós-graduandos, um inovador espaço de contato direto com realidades concretas, de desenvolvimento de habilidades e de aplicação de conhecimentos, numa interação de saberes acadêmicos e populares, tomando como tema a saúde, a alimentação e a qualidade de vida.

Através da metodologia aplicada, busca-se elevar os níveis de segurança dos alimentos comercializados na CUASO, envolvendo um processo equilibrado entre inocuidade, qualidade de visa, prosperidade econômica dos comerciantes, cumprimento da legislação, bem estar e sustentabilidade socioambiental dos usuários do campus.

\section{REFERÊNCIAS BIBLIOGRÁFICAS}

[1] CAMPOS, E. de S. Cidade Universitária da Universidade de São Paulo: Aspectos Gerais do Planejamento e Execução. São Paulo: Comissão da Cidade Universitária, 1954.

[2] CONSEA (Conselho Nacional de Segurança Alimentar e Nutricional). Relatório Final da II Conferência Nacional de Segurança Alimentar e Nutricional. Pernambuco, 2004. Disponível em: http://www4.planalto.gov.br/consea/publicacoes/ publiucacoes-arquivos/relatorio-final-ii-conferencia-nacional-de-seguranca-alimentar-e-nutricional. Acesso em: 27 jul. 2012.

[3] EL ANDALOUSSI, K. Pesquisas-ações: ciências, desenvolvimento, democracia. Trad. Michel Thiollent. São Carlos: EdUFSCar, 2004.

[4] INSTRUÇÃO NORMATIVA N. 62, de 26 de agosto de 2003. Métodos Analíticos Oficiais para Analises Microbiológicas para Controle de Produtos de Origem Animal e Água. Diário Oficial da República Federativa do Brasil, 18 set. 2003, Seção 1, p. 14.

[5] PODER EXECUTIVO do Estado de São Paulo. Lei n. 10083, de 23 de setembro de 1998. Dispõe sobre o Código Sanitário Estadual. Diário Oficial da União, 24 set. 1998.

[6] PREFEITURA DO MUNICÍPIO de São Paulo. Lei Municipal n. 13725, de 9 de janeiro de 2004. Dispõe sobre o Código Sanitário do Município de São Paulo. 
[7] Manual de Boas Práticas de Manipulação de Alimentos. São Paulo: Secretaria Municipal de Saúde/Coordenação de Vigilância em Saúde, 2006.

[8] RDC n. 216, de 15 de setembro de 2004. Dispõe sobre Regulamento Técnico de Boas Práticas para Serviços de Alimentação. Diário Oficial da República Federativa do Brasil, 16 set. 2003 .

[9] SECRETARIA DE ESTADO da Saúde. Portaria CVS-6/99, de 10 de março de 1999, alterada pela CVS 18, de o9 de setembro de 2008. Regulamento Técnico, que estabelece os Parâmetros e Critérios para o Controle Higiênico-Sanitário em Estabelecimentos de Alimentos.

[10] SECRETARIA MUNICIPAL DE SAÚDE de São Paulo. Portaria SMS n. 2.619, de 06 de dezembro de 2011. Manual de boas práticas.

[11] UNIVERSIDADE DE SÃO PAULO. USP em Números 2012 - Base de dados 2011. Disponível em: https://uspdigital.usp.br/anuario/tabelas/usp_em_numeros.pdf?codmnu=2786. Acesso em: 27 jul. 2012.

BRUNO AUGUSTI PORFÍRIO, DANIELA NOGUEIRA MALIZIA, EVELISE OLIVEIRA TELLES, RAFAEL ALMEIDA FERREIRA DE ABREU, RENAN TRIPODE BATARQUINI, SIMONE DE CARVALHO BALIAN, TATHIANA IURI HANASILO E THAÍS HELENA NISHIKATA DE OLIVEIRA Faculdade de Medicina Veterinária e Zootecnia da Universidade de São Paulo (V) - Av. Prof. Dr. Orlando Marques de Paiva, 87 - Cidade Universitária - CEP 05508270 - São Paulo-SP - e-mail:balian@usp.br

MARIA CRISTINA DE LOURDES GUARNIERI E RICARDO PRIST Prefeitura do Campus USP da Capital (PUSP-C). 